\title{
(2) OPEN ACCESS \\ Needs assessment of resuscitative endovascular balloon occlusion of the aorta (REBOA) in patients with major haemorrhage: a cross-sectional study
}

\author{
Bård Neuenkirchen Godø (ㄷ) , Jostein Rodseth Brede 주, 2,3,4,5 \\ Andreas Jorstad Krüger ${ }^{3,4,5}$
}

\begin{abstract}
Handling editor Ed Benjamin Graham Barnard

${ }^{1}$ Faculty of Medicine and Health Sciences, Norwegian University of Science and Technology, Trondheim, Norway 2Department of Anesthesiology and Intensive Care Medicine, St Olavs Hospital University Hospital in Trondheim, Trondheim, Norway ${ }^{3}$ Department of Research and Development, Norwegian Air Ambulance Foundation, Oslo, Norway

${ }^{4}$ Department of Emergency Medicine and Pre-Hospital Services, St Olavs Hospital University Hospital in Trondheim Trondheim, Norway ${ }^{5}$ Department of Circulation and Medical Imaging, Norwegian University of Science and Technology Faculty of Medicine and Health Sciences, Trondheim, Sør-Trøndelag, Norway
\end{abstract}

Correspondence to Bård Neuenkirchen Godø, Norwegian University of Science and Technology Faculty of Medicine and Health Sciences, 7030 Trondheim, Norway; baardng@stud.ntnu.no

Received 23 October 2020 Revised 15 April 2021 Accepted 25 April 2021

\section{ABSTRACT}

Background Resuscitative endovascular balloon occlusion of the aorta (REBOA) can be used as an adjunct treatment in traumatic abdominopelvic haemorrhage, ruptured abdominal aortic aneurysms, postpartum haemorrhage (PPH), gastrointestinal bleeding and iatrogenic injuries during surgery. This needs assessment study aims to determine the number of patients eligible for REBOA in a typical Norwegian population.

Methods This was a retrospective cross-sectional study based on data obtained from blood bank registries and the Norwegian Trauma Registry for the years 2017-2018. Patients who received $\geq 4$ units of packed red blood cells (PRBCs) within 6 hours and met the anatomical criteria for REBOA or patients with relevant Abbreviated Injury Scale codes with concurrent hypotension or transfusion of $\geq 4$ units of PRBCs within 6 hours were identified. A detailed two-step chart review was performed to identify potentially eligible REBOA candidates. Descriptive data were collected and compared between subgroups using non-parametric tests for statistical significance.

Results of 804 patients eligible for inclusion, 53 patients were regarded as potentially REBOA eligible (corresponding to 5.7 per 100000 adult population/ year). Of these, 19 actually received REBOA. Among the identified eligible patients, 44 (83\%) had a nontraumatic aetiology. Forty-two patients (79\%) were treated at a tertiary care hospital. Fourteen (78\%) of the REBOA procedures were due to $\mathrm{PPH}$.

Conclusion The number of patients potentially eligible for REBOA after haemorrhage is low, and most cases are non-traumatic. Most patients were treated at a tertiary care hospital. The exclusion of non-traumatic patients results in a substantial underestimation of the number of potentially REBOA-eligible patients.

\section{INTRODUCTION}

Haemorrhage is the leading cause of death in trauma patients during the first 24 hours after a trauma incident and the second largest cause of prehospital deaths after trauma to the central nervous system. ${ }^{12}$ Exsanguination is also a feared outcome of ruptured aortic aneurysms and postpartum haemorrhage (PPH). ${ }^{34}$

Resuscitative endovascular balloon occlusion of the aorta (REBOA) allows effective haemorrhage control in non-compressible abdominal and pelvic bleeding. ${ }^{6}$ The procedure is used as a bridge

\section{Key messages}

What is already known on this subject

- Resuscitative endovascular balloon occlusion of the aorta (REBOA) can be used to manage noncompressible torso haemorrhage.

- REBOA-eligible patients include those with traumatic abdominopelvic haemorrhage, ruptured abdominal aortic aneurysm and others (eg, postpartum haemorrhage).

- Multiple studies have assessed the potential use of REBOA following trauma, but none have assessed the potential use in non-trauma populations.

What this study adds

- Interrogation of blood bank registries is an effective method to identify non-trauma REBOA-eligible patients.

- The overall incidence of REBOA-eligible patients is low, and over three-quarters have a nontrauma aetiology.

to definite treatment in exsanguinating trauma patients, and its application outside the hospital has recently been assessed. ${ }^{78}$

In 2019, the first Delphi process on REBOA was published to clarify indications. ${ }^{9}$ However, there is still no widespread consensus on the indications for REBOA in trauma care. A systematic review proposed that patients eligible for REBOA could be divided into three main groups: those with traumatic abdominopelvic haemorrhage, those with ruptured abdominal aortic aneurysms and those with other causes, including $\mathrm{PPH}$, bleeding from the gastrointestinal tract and extensive bleeding during pelvic surgery. ${ }^{3}$ The authors reported that 90\% of patients treated with REBOA due to trauma had established haemorrhagic shock (systolic blood pressure below $90 \mathrm{~mm} \mathrm{Hg}$ ).

Any new treatment modality should ideally be implemented based on a thorough analysis of its expected effectiveness, associated risks and costbenefit ratio. It is currently not known if REBOA improves survival in any patient cohort, ${ }^{3} 1011$ but ongoing studies will try to assess the effect of REBOA in trauma care. ${ }^{12}$ Multiple studies have assessed the need for REBOA in traumatic populations. ${ }^{13-16}$ However, there are no studies focusing 
on non-traumatic indications, resulting in a critical gap in the literature.

As such, the first aim was to assess the number of patients, traumatic and non-traumatic, eligible for REBOA in a typical Norwegian population. The second aim was to describe these patients' demographics, physiology and fluid resuscitation.

\section{METHODS}

\section{Study setting}

We performed a retrospective cross-sectional study based on patient data obtained from two data registries: the Norwegian Trauma Registry (NTR) and local hospital blood bank registries in central Norway. We included patients admitted to hospitals in the 2-year period from 1 January 2017 to 31 December 2018.

The hospital system in the geographical area under study comprises seven hospitals situated in Trondheim, Molde, Kristiansund, Orkdal, Levanger, Namsos and Tynset. St. Olavs University Hospital in Trondheim is a tertiary care hospital $(\mathrm{TCH})$ and a major trauma centre, and the others are acute care hospitals (ACH). The area is mixed urban and rural, with an adult population (18 years or older) of approximately $468000 .^{17}$ The city of Trondheim and its surrounding area is regarded as urban. ${ }^{18}$ Currently, REBOA, as an in-hospital treatment, is routinely performed only by interventional radiologists at the $\mathrm{TCH}$ for PPH. ${ }^{4}$ However, individual surgeons at the ACHs may by chance be trained in the technique and can perform REBOA when indicated. Some patients were transferred from an $\mathrm{ACH}$ to the TCH. The level of care was recorded as the hospital that the patient initially presented to.

Prehospital critical care is provided by a two-tier system consisting of paramedics and primary care physicians who are on-call 24/7 (tier one) and four anaesthesiologist-staffed prehospital mobile units (tier two).

Because we expected a small annual number of REBOAeligible patients, a 2-year period was chosen. The NTR is a national quality register hosted by Oslo University Hospital. These data are registered in a central database (Medical Registry System) that ensures data security and integrity. Trauma patients in the NTR are registered manually by local registrars. All registrars are certified and have completed specific coding courses.

All hospitals except one, Kristiansund Hospital, reported to the NTR during the period. Kristiansund Hospital is not intended to receive trauma patients as it is not included as a trauma-receiving hospital within the regional trauma system.

\section{Data collection and processing}

Patients were identified via interrogation of both the NTR and individual hospital blood bank registries (figure 1).

\section{Trauma cohort}

Adult patients (18 years or older) registered in the NTR with an Abbreviated Injury Scale (AIS) code corresponding to a highgrade abdominopelvic injury were identified. We used the same anatomical indications for trauma patients as those published in earlier needs assessments of REBOA (table 1). ${ }^{13-15}$

Patients with a registered systolic blood pressure (SBP) $<90 \mathrm{~mm}$ $\mathrm{Hg}$ or transfusion of four or more units of packed red blood cells (PRBC) within 6 hours, irrespective of admission, were included in the subsequent chart reviews. Non-compressible haemorrhage in the superior mediastinum, axilla, neck or face and aortic dissection are all regarded as contraindications to REBOA treatment. Patients with these contraindications were excluded.

\section{Blood cohort}

Data were obtained from the blood bank registry at each hospital. Adult patients (18 years or older) who were transfused with four or more units of PRBC in 6 hours were identified. PRBC is a unit of $200-300 \mathrm{~mL}$ erythrocyte concentrate with saline, adenosine, glucose and mannitol additive. Patients with anatomical indications for REBOA (table 1) were included in the subsequent chart review. Patients with the same contraindications as those used in the trauma cohort were excluded.

\section{Processing}

Duplicates in the two cohorts were removed. A primary chart review was performed by the first author (BNG) to exclude patients who were clearly not eligible for REBOA, those with stable cardiovascular physiology and those with distinct responses to crystalloid or blood resuscitation. The remaining patients were then subjected to a secondary chart review by two experienced consultant anaesthesiologists (JRB and AJK). All documentation on the acute incident registered in the electronic patient journal was assessed. The inclusion criteria were based on a consensus regarding indications from 2019 ${ }^{9}$ : anatomical indications for REBOA (table 1), commencement of a massive transfusion protocol or transfusion of four or more units of blood products during the acute incident and patients registered as haemodynamically unstable in the patient's medical record by the patient-responsible anaesthesia personnel or when organs were removed to enable surgical haemostasis. Patients who received REBOA or in whom REBOA was deemed necessary by the involved healthcare providers were also included. Patients with a high burden of comorbidities, those with a poor quality of life and patients in a state regarded as futile for treatment were excluded. Those patients evaluated as eligible by both reviewers were confirmed as eligible REBOA candidates. Disputed patient cases were discussed until agreement.

For eligible REBOA candidates, we extracted the lowest registered SBP prior to treatment, heart rate at the lowest registered SBP, total volumes of fluids, hospital, age, sex, survival status (dead/alive) until discharge, site of bleeding and AIS codes. The number of fluid transfusions reported during the acute incidence was defined as those administered during the period from injury or the start of decompensation until definitive treatment or death. Any difference in fluid resuscitation between $\mathrm{TCH}$ and $\mathrm{ACH}$ was assessed.

\section{STATISTICAL ANALYSIS}

Statistical analysis was performed with SPSS, V.25.0 (2017) (IBM Corporation, Armonk, New York, USA). Continuous variables are reported as medians with IQRs. Categorical variables are described as counts and/or proportions (\%). Incidence is presented as the rate with $95 \%$ CI. Comparisons of medians between groups with non-normal distribution of data were performed with the Mann-Whitney $U$ test. Comparisons of proportions were performed with the Fisher's exact test. A P value of $<0.05$ was regarded as statistically significant.

\section{ETHICAL REVIEW}

A Data Protection Impact Assessment was performed to identify and minimise the data protection risks of the study.

\section{Patient and public involvement}

This study was conducted without direct patient involvement. The patient's and public's interests were maintained by the involvement of Data Protection Officers of local health trusts. 


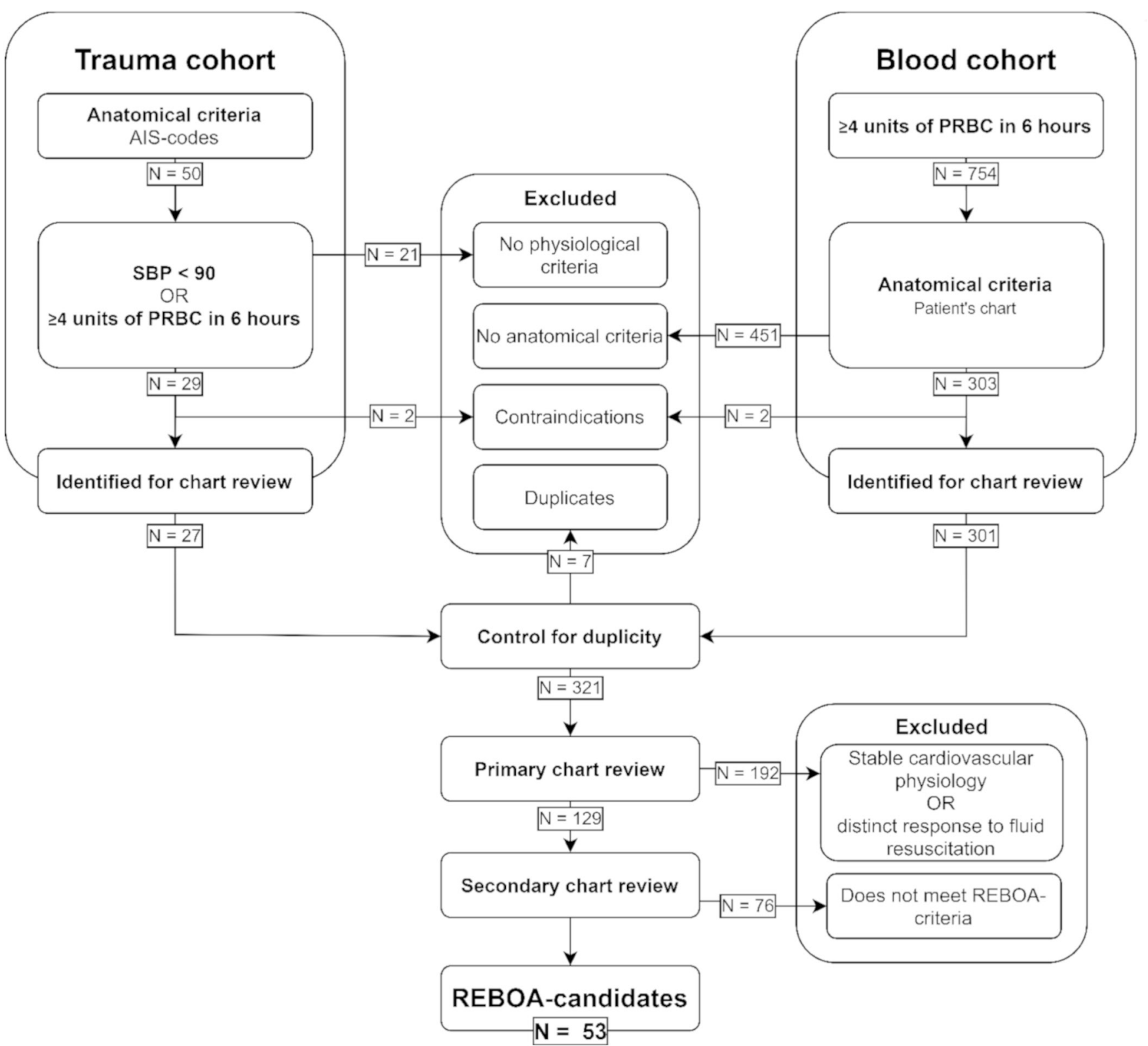

Figure 1 Flow chart of patient selection. Patients were identified through two different algorithm-based cohorts, which were followed by a twostep chart review. AIS, Abbreviated Injury Scale; PRBC, packed red blood cells; SBP, systolic blood pressure; REBOA, resuscitative endovascular balloon occlusion of the aorta.

Table 1 Site or type of haemorrhage considered eligible for REBOA

\begin{tabular}{ll}
\hline Groups & \\
\hline Traumatic & Non-traumatic \\
\hline High grade injury to & Ruptured abdominal aortic aneurysm \\
\hline Liver & Postpartum haemorrhage \\
\hline Kidney & Gastrointestinal haemorrhage \\
\hline Spleen & latrogenic injury during \\
\hline Named abdominal vessel & Pelvic surgery \\
\hline Named pelvic vessel & Cancer surgery \\
\hline Mesenteric disruption & Vascular surgery \\
\hline Pelvic fracture with ring disruption & \\
\hline Traumatic amputation at or near the hip & \\
\hline
\end{tabular}

An Abbreviated Injury Scale score $\geq 3$ was regarded as a high-grade injury.

REBOA, resuscitative endovascular balloon occlusion of the aorta.

\section{RESULTS}

Initially, we identified 804 cases in the two cohorts (figure 1). The blood cohort consisted of 754 patients who received four or more units of PRBC in 6 hours. Of these, 303 had anatomical indications for REBOA. The trauma cohort consisted of 50 patients with anatomical indications for REBOA. Of these, 29 fulfilled the physiological criteria for REBOA. Four patients, two from each cohort, were excluded because of aortic dissections. Seven patients were found in both cohorts. Duplicates were removed from further analyses. A total of 321 patients were evaluated in the primary chart review. One hundred and ninety-two patients were regarded as clearly not eligible in this phase, leaving 129 patients to be evaluated in the secondary chart review. This process resulted in 53 patients being regarded as potentially REBOA eligible (table 2 ). 
Table 2 Demographics, presenting physiology, fluid resuscitation, level of care, type of haemorrhage and mortality in potentially eligible REBOA candidates

\begin{tabular}{|c|c|c|c|}
\hline & Total & Subgroups & \\
\hline & REBOA candidates & Trauma & Non-trauma \\
\hline$n$ & $53(100.0)$ & $9(17.0)$ & $44(83.0)$ \\
\hline \multicolumn{4}{|l|}{ Demographics } \\
\hline \multicolumn{4}{|l|}{ Gender, n (\%) } \\
\hline Female & $30(56.6)$ & $2(22.2)$ & $28(63.6)$ \\
\hline Male & $23(43.4)$ & $7(77.8)$ & $16(36.4)$ \\
\hline Age, median years (IQR) & $45(32-69)$ & $54(42-64)$ & $38(31-71)$ \\
\hline \multicolumn{4}{|l|}{ Presenting physiology, median (IQR) } \\
\hline SBP, mm Hg (lowest registered) & $65(50-80)$ & $51(41-70)$ & $70(51-80)$ \\
\hline $\mathrm{HR}$, per min (at time of lowest registered SBP) & $100(87-120)$ & $106(102-140)$ & $99(81-120)$ \\
\hline \multicolumn{4}{|l|}{ Fluid resuscitation, median $\mathrm{mL}$ (IQR) } \\
\hline PRBC & $2000(1500-3500)$ & $3500(1500-4625)$ & $1875(1500-2750)$ \\
\hline Plasma & $1200(800-2200)$ & $1800(1000-3600)$ & $1200(800-2000)$ \\
\hline Platelets & $500(250-750)$ & $750(375-1125)$ & $500(250-500)$ \\
\hline Crystalloids & $3000(2000-4382)$ & $3000(2500-3900)$ & $3000(2000-4766)$ \\
\hline PRBC in 6 hours, median units (IQR) & $8(6-15)$ & $12(6-18)$ & $7(6-12)$ \\
\hline \multicolumn{4}{|l|}{ Level of care, n (\%) } \\
\hline TCH & $42(79.2)$ & $7(77.8)$ & $35(79.5)$ \\
\hline $\mathrm{ACH}$ & $11(20.8)$ & $2(22.2)$ & $9(20.5)$ \\
\hline \multicolumn{4}{|l|}{ Type of haemorrhage, $n$ (\%) } \\
\hline Spleen & $2(3.8)$ & $2(22.2)$ & \\
\hline Mesenteric disruption & $2(3.8)$ & $2(22.2)$ & \\
\hline Pelvic fracture with ring disruption & $5(9.4)$ & $5(55.6)$ & \\
\hline Ruptured abdominal aortic aneurysm & $9(17.0)$ & & $9(20.5)$ \\
\hline Gastrointestinal haemorrhage & $6(11.3)$ & & $6(13.6)$ \\
\hline Postpartum haemorrhage & $23(43.4)$ & & $23(52.3)$ \\
\hline latrogenic injury & $6(11.3)$ & & $6(13.6)$ \\
\hline Dead before discharge, $n(\%)$ & $11(20.8)$ & $1(11.1)$ & $10(22.7)$ \\
\hline
\end{tabular}

One unit of PRBC contains $200-300 \mathrm{~mL}$, one unit of plasma contains $200 \mathrm{~mL}$ and one unit of platelets (from eight donors or apheresis from one donor) contains $180-350 \mathrm{~mL}$

ACH, acute care hospital; HR, heart rate; PRBCs, packed red blood cells; REBOA, resuscitative endovascular balloon occlusion of the aorta; SBP, systolic blood pressure (lowest registered during acute incident); $T$ TCH, tertiary care hospital.

There were no differences in physiological parameters, or the volumes of fluids used in resuscitation between the group of potentially eligible patients who received REBOA and those who did not (table 3 ).

The TCH and $\mathrm{ACH}$ fluid resuscitation conventions differed only regarding platelets, with median values of $500 \mathrm{~mL}$ and $250 \mathrm{~mL}$, respectively $(\mathrm{p}=0.029)$. There were no significant differences in the provision of PRBC, plasma or crystalloids (table 4).

Fifty-three patients with a REBOA-amenable injury or condition in an adult population of 468000 indicates an estimated incidence of 5.7 (95\% CI 4.3 to 7.4) per 100000 adult population/year. Of the 2210 trauma patients in the area over the 2-year period, $0.4 \%(n=9)$ were regarded as potentially eligible for REBOA as an adjunct treatment.

\section{DISCUSSION}

This study finds that most patients eligible for REBOA as an adjunct treatment have non-traumatic aetiology. This is perhaps in contrast to current practice, where seemingly traumatic aetiology dominates. Our results estimate an incidence of 5.7 per 100000 adult population/year.

Approximately one-third of the REBOA-eligible patients actually received REBOA, and most of these were due to PPH. This may reflect a specifically good collaboration between interventional radiologists and gynaecologists at the $\mathrm{TCH} .{ }^{4}$ Regardless, it indicates that non-traumatic aetiology should not be overlooked. Patients registered at the TCH might have been eligible for REBOA earlier in the chain of care, during transfer, at an $\mathrm{ACH}$, or even prehospital if it had been available.
Table 3 Comparison of the subgroups of potentially REBOA-eligible patients who did and did not receive REBOA

\begin{tabular}{|c|c|c|c|}
\hline & \multicolumn{2}{|l|}{ REBOA conducted } & \multirow[b]{2}{*}{$P$ value } \\
\hline & Yes & No & \\
\hline$n,(\%)$ & $19(35.8)$ & $34(64.2)$ & \\
\hline \multicolumn{4}{|l|}{ Level of care, $\mathrm{n}(\%)$} \\
\hline $\mathrm{TCH}$ & $18(94.7)$ & $25(73.5)$ & \\
\hline $\mathrm{ACH}$ & $1(5.3)$ & $9(26.5)$ & \\
\hline \multicolumn{4}{|l|}{ Physiology, median (IQR) } \\
\hline $\mathrm{SBP}, \mathrm{mm} \mathrm{Hg}$ & $70(60-88)$ & $63(49-79)$ & 0.130 \\
\hline$H R$, per min & $103(83-117)$ & $99(87-130)$ & 0.866 \\
\hline \multicolumn{4}{|l|}{$\begin{array}{l}\text { Fluid resuscitation, median } \\
\mathrm{mL}(\mathrm{IQR})\end{array}$} \\
\hline PRBC & $2250(1500-3500)$ & $1875(1438-3688)$ & 0.752 \\
\hline Plasma & $1200(800-2000)$ & $1300(800-2400)$ & 0.985 \\
\hline Platelets & $500(250-500)$ & $500(250-813)$ & 0.586 \\
\hline Crystalloids & $3770(2050-5093)$ & $2950(2000-4000)$ & 0.275 \\
\hline \multicolumn{4}{|l|}{ Type of haemorrhage, $n(\%)$} \\
\hline PPH & $14(73.6)$ & $9(26.6)$ & \\
\hline rAAA & $3(15.8)$ & $6(17.6)$ & \\
\hline Trauma & $1(5.3)$ & $8(23.5)$ & \\
\hline GI & $1(5.3)$ & $5(14.7)$ & \\
\hline latrogenic & $0(0.0)$ & $6(17.6)$ & \\
\hline Dead before discharge, $\mathrm{n}(\%)$ & $3(15.8)$ & $8(23.5)$ & 0.726 \\
\hline
\end{tabular}

One patient received REBOA at the TCH after being transferred from an ACH. This patient's level of care is registered as ACH in tables 2 and 4 , and as TCH in table 3.

$\mathrm{ACH}$, acute care hospital; $\mathrm{Gl}$, gastrointestinal; $\mathrm{HR}$, heart rate; $\mathrm{PPH}$, postpartum haemorrhage; $\mathrm{PRBCs}$, packed red blood cells; rAAA, ruptured abdominal aortic aneurysm; REBOA, resuscitative endovascular balloon occlusion of the aorta; SBP, systolic blood pressure; TCH, tertiary care hospital. 


\begin{tabular}{|c|c|c|c|}
\hline & \multicolumn{2}{|l|}{ Level of care } & \multirow[b]{2}{*}{$P$ value } \\
\hline & TCH & $\mathrm{ACH}$ & \\
\hline n, (\%) & $42(79.2)$ & $11(20.8)$ & \\
\hline \multicolumn{4}{|c|}{ Fluid resuscitation, median mL (IQR) } \\
\hline PRBC & $1875(1438-4250)$ & $2000(1500-3500)$ & 0.974 \\
\hline Plasma & $1400(800-2550)$ & $1200(600-1800)$ & 0.059 \\
\hline Platelets & $500(250-813)$ & $250(0-500)$ & 0.029 \\
\hline Crystalloids & $3290(1727-4000)$ & $3000(2000-4500)$ & 0.879 \\
\hline
\end{tabular}

$\mathrm{ACH}$, acute care hospital; PRBCs, packed red blood cells; $\mathrm{TCH}$, tertiary care hospital.

Norwegian data show a low incidence of life-threatening trauma as well as low severity after trauma. ${ }^{18}$ Hence, our results may not translate to larger cities or urban areas where the trauma burden is more severe. However, we believe this study provides a realistic assessment of need in our area, as well as other Scandinavian or European areas with similar population densities. For example, we found the same estimation of trauma patients to be potentially REBOA eligible as a recent Swedish needs assessment. ${ }^{16}$ The design of our study was based on previously published studies, which focused solely on traumatic patients even though the literature describes other utilisations for the procedure. ${ }^{39}$ By adding the use of blood transfusions as a primary input, we were able to identify patients with nontraumatic indications for REBOA, and this greatly increased the number of REBOA candidates.

One needs assessment at a major trauma centre reported that $45 \%$ of the patients identified through an algorithm with anatomical and physiological criteria were regarded as truly REBOA eligible after chart review. ${ }^{14}$ In comparison, we found that only $17 \%$ were eligible. We believe that the use of blood transfusions as a primary input increases the necessity of a subsequent chart review, and in contrast to Dumas et al, ${ }^{15}$ we describe the inclusion/exclusion criteria of these reviews. One can argue that disputed patients who were initially regarded as eligible by only one investigator might not be clear-cut REBOA candidates. However, the subsequent joint chart review of these patients provides a plausible basis to regard them as eligible. It also demonstrates the complexity of real-life decision making in these patients.

We did not observe any difference in physiology or fluid resuscitation between those that did and did not receive REBOA. This increases the plausibility that all these patients were REBOA eligible. There was a small, non-significant difference in survival between the group that received REBOA and the group that did not. There was no difference in volumes of fluids used in resuscitation at $\mathrm{ACH}$ and the $\mathrm{TCH}$, except for transfusion of platelets. This is most likely due to differences in the availability of platelets between hospitals. Also, we observed that a high volume of crystalloids was used relative to blood transfusions.

The detection of possible differences in factors like postoperative morbidity, length of surgery, length of stay at hospital or the need to remove organs to enable surgical haemostasis between the two groups was beyond the scope of this study. Such outcome measures, combined with survival and fluid resuscitation requirements, would probably be the best endpoint in future studies.

REBOA is a highly invasive procedure that demands technical skill and is only achievable through considerable practice in the Seldinger technique and the use of ultrasound. ${ }^{8}{ }^{19}{ }^{20} \mathrm{~A}$ training programme designed for prehospital anaesthesiologists has been previously reported. ${ }^{21}$ Our findings suggest that at ACHs, fewer than one patient per year is potentially REBOA eligible. There might therefore be a need for REBOA competence at TCHs, but our findings question the need for REBOA competence at ACHs. However, our study does not include potential cases where REBOA could have been used in out-of-hospital non-traumatic cardiac arrests or patients eligible for early femoral arterial access. ${ }^{22-24}$

\section{LIMITATIONS}

This study has several limitations. First, the specificity of the algorithm used to identify REBOA candidates decreases when a blood cohort is added, increasing the necessity of chart review. Second, we admit that the chart review would have been more reliable if it was performed by reviewers not directly involved in the study. However, we report the inclusion/exclusion criteria that were used to ensure a transparent decision process. Third, the volumes of fluids reported were extracted from the charts and blood bank registry. These values were not always in agreement, possibly due to the use of emergency blood immediately available in the resuscitation room or errors in registration. When different values were found, the highest value was reported as we regarded lack of registration as more likely than too many registrations. Finally, manual registrations in the NTR allows for erroneous inputs. However, due to certifications and specific coding courses, the numbers of mistakes expected in the data input are low.

\section{CONCLUSIONS}

This analysis of national trauma registry data and the novel use of blood bank data constitutes the first needs assessment of REBOA including non-traumatic aetiology, as well as the first needs assessment of REBOA in patients with major haemorrhage in Norway ${ }^{24}$. The number of patients eligible for REBOA after haemorrhage is low, and most cases are non-traumatic. We find that excluding non-traumatic patients greatly underestimates the number of potentially REBOA-eligible patients. This finding is important for future implementation of REBOA in clinical practice.

Correction notice This article has been corrected since it was published Online first. Figure 1 was showing an error in the online version; this was corrected.

Twitter Jostein Rodseth Brede @BredeJostein and Andreas Jorstad Krüger @ andreasjkruger

Acknowledgements We would like to thank the members of the Norwegian Trauma Registry, the information and technology administrators of the blood banks, the involved Data Protection Officers, Nord-Trøndelag Hospital Trust, Møre og Romsdal Hospital Trust, Innlandet Hospital Trust and St. Olav Hospital Trust.

Contributors JRB and AJK conceived the study idea. All authors contributed to the design of the study. BNG collected the data and performed the analyses. All authors discussed the results and contributed to the final manuscript.

Funding This study was funded by the Norwegian University for Science and Technology. The contributions from other personnel were agreed on without any financial compensation.

Competing interests None declared.

Patient and public involvement Patients and/or the public were not involved in the design, or conduct, or reporting, or dissemination plans of this research.

Patient consent for publication Not required.

Ethics approval The study was approved by the Regional Committees for Medical and Health Research Ethics (reference 2019/793/REK-Midt) and the Data Protection Officer in every health trust was involved.

Provenance and peer review Not commissioned; externally peer reviewed. 
Data availability statement Data are available on reasonable request. Data were obtained from blood bank registries, the Norwegian Trauma Registry and the electronic patient journal of Central Norway Regional Health Authority. The dataset consists of deidentified participant data. Because of the interconnection of data from multiple registries, the Norwegian Center for Research Data has concluded that the dataset in whole could indirectly identify specific patients. Therefore, the data are stored on protected servers owned by St. Olav Hospital Trust and is not made publicly open.

Open access This is an open access article distributed in accordance with the Creative Commons Attribution Non Commercial (CC BY-NC 4.0) license, which permits others to distribute, remix, adapt, build upon this work non-commercially, and license their derivative works on different terms, provided the original work is properly cited, appropriate credit is given, any changes made indicated, and the use is non-commercial. See: http://creativecommons.org/licenses/by-nc/4.0/.

\section{ORCID iDs}

Bård Neuenkirchen Godø http://orcid.org/0000-0001-7337-5274

Jostein Rodseth Brede http://orcid.org/0000-0001-9497-1147

\section{REFERENCES}

1 Kauvar DS, Lefering R, Wade CE. Impact of hemorrhage on trauma outcome: an overview of epidemiology, clinical presentations, and therapeutic considerations. J Trauma 2006;60:S3-11.

2 Davis JS, Satahoo SS, Butler FK, et al. An analysis of prehospital deaths: who can we save? J Trauma Acute Care Surg 2014;77:213-8.

3 Borger van der Burg BLS, van Dongen TTCF, Morrison JJ, et al. A systematic review and meta-analysis of the use of resuscitative endovascular balloon occlusion of the aorta in the management of major exsanguination. Eur J Trauma Emerg Surg 2018;44:535-50.

4 Stensaeth KH, Sovik E, Haig INY, et al. Fluoroscopy-free resuscitative endovascular balloon occlusion of the aorta (REBOA) for controlling life threatening postpartum hemorrhage. PLoS One 2017;12:e0174520.

5 Stannard A, Eliason JL, Rasmussen TE. Resuscitative endovascular balloon occlusion of the aorta (REBOA) as an adjunct for hemorrhagic shock. J Trauma 2011;71:1869-72.

6 Brenner ML, Moore LJ, DuBose JJ, et al. A clinical series of resuscitative endovascular balloon occlusion of the aorta for hemorrhage control and resuscitation. J Trauma Acute Care Surg 2013;75:506-11.

7 Thabouillot O, Bertho K, Rozenberg E, et al. How many patients could benefit from REBOA in prehospital care? A retrospective study of patients rescued by the doctors of the Paris fire Brigade. J R Army Med Corps 2018;164:267-70.

8 Brede JR, Lafrenz T, Klepstad P, et al. Feasibility of pre-hospital resuscitative endovascular balloon occlusion of the aorta in non-traumatic out-of-hospital cardiac arrest. J Am Heart Assoc 2019;8:e014394.

9 Borger van der Burg BLS, Kessel B, DuBose JJ, et al. Consensus on resuscitative endovascular balloon occlusion of the aorta: a first consensus paper using a Delphi method. Injury 2019;50:1186-91.
10 Karkos CD, Papadimitriou CT, Chatzivasileiadis TN, et al. The impact of aortic occlusion balloon on mortality after endovascular repair of ruptured abdominal aortic aneurysms: a meta-analysis and meta-regression analysis. Cardiovasc Intervent Radiol 2015;38:1425-37.

11 Morrison JJ, Morrison JJ, Galgon RE, et al. A systematic review of the use of resuscitative endovascular balloon occlusion of the aorta in the management of hemorrhagic shock. J Trauma Acute Care Surg 2016;80:324-34.

12 ISRCTN - ISRCTN16184981: The effectiveness and cost-effectiveness of Resuscitative Endovascular Balloon Occlusion of the Aorta (REBOA) for trauma.

13 Morrison JJ, Ross JD, Rasmussen TE, et al. Resuscitative endovascular balloon occlusion of the aorta: a gap analysis of severely injured UK combat casualties. Shock 2014;41:388-93.

14 Barnard EBG, Morrison JJ, Madureira RM, et al. Resuscitative endovascular balloon occlusion of the aorta (REBOA): a population based gap analysis of trauma patients in England and Wales. Emerg Med J 2015;32:926-32.

15 Dumas RP, Holena DN, Smith BP, et al. Resuscitative endovascular balloon occlusion of the aorta: assessing need in an urban trauma center. J Surg Res 2019;233:413-9.

16 Mill V, Wellme E, Montán C. Trauma patients eligible for resuscitative endovascular balloon occlusion of the aorta (REBOA), a retrospective cohort study. Eur J Trauma Emerg Surg 2020. doi:10.1007/s00068-020-01345-w. [Epub ahead of print: 23 Mar 2020].

17 StatBank Norway. [Dataset] Statistics Norway: 07459: Population, by region, age, contents and year, 2018. Available: https://www.ssb.no/en/statbank/table/07459/ tableViewLayout2/ [Accessed 13 Feb 2020].

18 Uleberg 0, Kristiansen T, Pape K, et al. Trauma care in a combined rural and urban region: an observational study. Acta Anaesthesio/ Scand 2017;61:346-56.

19 Borger van der Burg BLS, Hörer TM, Eefting D, et al. Vascular access training for REBOA placement: a feasibility study in a live tissue-simulator hybrid porcine model. J R Army Med Corps 2019;165:147-51.

20 Brenner M, Bulger EM, Perina DG, et al. Joint statement from the American College of surgeons Committee on trauma (ACS cot) and the American College of emergency physicians (ACEP) regarding the clinical use of resuscitative endovascular balloon occlusion of the aorta (REBOA). Trauma Surg Acute Care Open 2018;3:e000154.

21 Brede JR, Lafrenz T, Krüger AJ, et al. Resuscitative endovascular balloon occlusion of the aorta (REBOA) in non-traumatic out-of-hospital cardiac arrest: evaluation of an educational programme. BMJ Open 2019;9:e027980.

22 Daley J, Morrison JJ, Sather J, et al. The role of resuscitative endovascular balloon occlusion of the aorta (REBOA) as an adjunct to ACLS in non-traumatic cardiac arrest. Am J Emerg Med 2017;35:731-6.

23 Osborn LA, Brenner ML, Prater SJ, et al. Resuscitative endovascular balloon occlusion of the aorta: current evidence. Open Access Emerg Med 2019;11:29-38.

24 Brede JR, Kramer-Johansen J, Rehn M. A needs assessment of resuscitative endovascular balloon occlusion of the aorta (REBOA) in non-traumatic out-of-hospital cardiac arrest in Norway. BMC Emerg Med 2020;20:28. 